\title{
Inhalt
}

Siglen $-X V I I$

Rudolf Meer, Giuseppe Motta, Gideon Stiening

Vom „Poison de l’imagination“ zur Essenz des Schematismus: Die

Einbildungskraft in der Philosophie, den Wissenschaften und den Künsten

des 17. und 18. Jahrhunderts - 1

\section{17. Jahrhundert}

Ursula Renz

Spinozist Cognitive Psychology: Spinoza's Concept of the Imagination - 9

Sarah Tropper

The Importance of Imagination in Leibniz -25

Hannes Fraissler

John Locke und wie man sich nicht vorstellt, eine andere Person zu sein -39

\section{18. Jahrhundert vor Kant}

Mischa von Perger

Voltaires Strategiewechsel beim Schildern der Einbildungskraft -61

Thomas Valentin Harb

Du Châtelet's Model of Imagination and Illusion -71

Andree Hahmann

Die Einbildungskraft eine „General-Kraft“? Mit Crusius zu den letzten Kräften der Seele -91

Udo Roth

Einbildungskraft und Verdauung. Zur medizinischen Sicht auf die Einbildungskraft bei Johann August Unzer — 115 
Paola Rumore

Imagination and Prejudice. Georg Friedrich Meier's Psychological

Understanding of Superstition -145

Hans-Peter Nowitzki

Christoph Martin Wieland und die Einbildungskraft -163

Gideon Stiening

Von Meisterköchen und „Fratzengesichtern“. J. G. H. Feders Theorie der

Einbildungskraft zwischen Erkennen und Wollen — 279

Simone De Angelis

Gedankenexperimente, Analogien und kühne Hypothesen. Die Bedeutung der ,Wissenschaften vom Leben' für die Beziehung von Anthropologie und Geschichtsdenken in der Spätaufklärung - ein programmatischer Entwurf - 303

\section{Kant und die Folgen}

\section{Radka Tomečková}

Zwischen Wahnsinn und Erkenntnistheorie. Die Einbildungskraft bei Locke, Hume und Kant -325

Corey W. Dyck

Imagination and Association in Kant's Theory of Cognition - 351

\section{Günter Zöller}

„Zum Raum wird hier die Zeit.“ Die Bildung der Zeit in Kants Kritik der reinen Vernunft 371

\section{Giuseppe Motta}

„bald rot, bald schwarz“. Die Zufälligkeit des Zinnobers im Kontext der Kantischen Theorie der Einbildungskraft — 391

\section{Rudolf Meer}

Eingebildete Gegenstände. Kants Konzept des focus imaginarius im Spannungsfeld der Träume eines Geistersehers und der Kritik der reinen Vernunft - 411 
Achim Vesper

Kant über Einbildungskraft und schöne Kunst — 435

Stefan Klingner

Das individuelle Subjekt. Zur Originalität von J. G. E. Maaß' Versuch über die Einbildungskraft — 453

Marion Heinz

Einbildungskraft und Antiintellektualismus. Zur Kritik von Heideggers Kantbuch -483

Schriftenverzeichnis 499

Personenregister $-\mathbf{5 0 7}$ 
